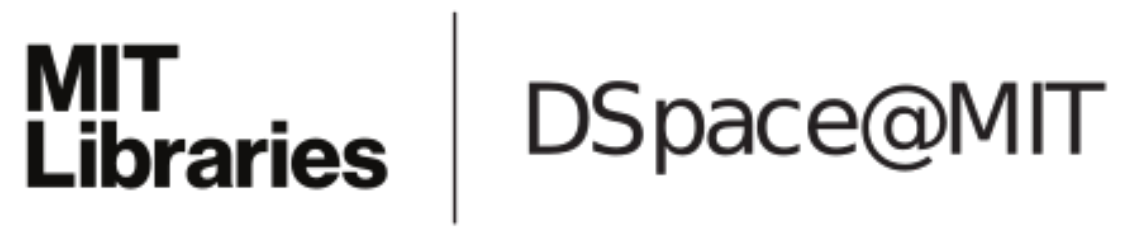

\author{
MIT Open Access Articles
}

Origins of the Unidirectional Spin Hall
Magnetoresistance in Metallic Bilayers

The MIT Faculty has made this article openly available. Please share how this access benefits you. Your story matters.

Citation: Avci, Can Onur et al. "Origins of the Unidirectional Spin Hall Magnetoresistance in Metallic Bilayers." Physical Review Letters 121, 8 (August 2018): 0827207 (c) 2018 American Physical Society

As Published: http://dx.doi.org/10.1103/PhysRevLett.121.087207

Publisher: American Physical Society

Persistent URL: http://hdl.handle.net/1721.1/117553

Version: Final published version: final published article, as it appeared in a journal, conference proceedings, or other formally published context

Terms of Use: Article is made available in accordance with the publisher's policy and may be subject to US copyright law. Please refer to the publisher's site for terms of use. 


\title{
Origins of the Unidirectional Spin Hall Magnetoresistance in Metallic Bilayers
}

\author{
Can Onur Avci, ${ }^{1}$ Johannes Mendil, ${ }^{2}$ Geoffrey S. D. Beach, ${ }^{1}$ and Pietro Gambardella ${ }^{2}$ \\ ${ }^{1}$ Department of Materials Science and Engineering, Massachusetts Institute of Technology, Cambridge, \\ Massachusetts 02139, USA \\ ${ }^{2}$ Department of Materials, ETH Zürich, 8093 Zürich, Switzerland
}

(Received 4 April 2018; published 24 August 2018)

\begin{abstract}
Recent studies evidenced the emergence of asymmetric electron transport in layered conductors owing to the interplay between electrical conductivity, magnetization, and the spin Hall or Rashba-Edelstein effects. Here, we investigate the unidirectional magnetoresistance (UMR) caused by the current-induced spin accumulation in $\mathrm{Co} / \mathrm{Pt}$ and $\mathrm{CoCr} / \mathrm{Pt}$ bilayers. We identify three competing mechanisms underpinning the resistance asymmetry, namely, interface and bulk spin-dependent electron scattering and electron-magnon scattering. Our measurements provide a consistent description of the current, magnetic field, and temperature dependence of the UMR and show that both positive and negative UMR can be obtained by tuning the interface and bulk spin-dependent scattering.
\end{abstract}

DOI: 10.1103/PhysRevLett.121.087207

The interconversion of charge and spin currents is a central theme in spintronics. The current-induced spin accumulation generated by the spin Hall effect (SHE) and/or the Rashba-Edelstein effect (REE) enables efficient magnetization switching, domain wall manipulation, and ferromagnetic resonance [1]. Moreover, the coupling between the spin and orbital moments of the charge carriers, as exemplified by the SHE and REE, is responsible for novel magnetoresistive phenomena, such as the spin Hall magnetoresistance (SMR) [2-10], the Hanle magnetoresistance [11,12], and the Rashba-Edelstein magnetoresistance (EMR) [13-20]. These phenomena are transforming our understanding of electric transport, leading to novel possibilities to sense the magnetization in devices. The archetypal SMR, e.g., arises from the conversion of a charge current density $\mathbf{j} \| \mathbf{x}$, flowing in the plane of a ferromagnetnormal-metal (FM/NM) bilayer, into a spin current diffusing along $\mathbf{z}$ into the FM with spin polarization $\boldsymbol{\sigma} \| \mathbf{y}$. For parallel or antiparallel orientation of $\boldsymbol{\sigma}$ and magnetization $\mathbf{m}$, part of the spin current is reflected at the FM/NM interface and backconverted into a charge current by the inverse SHE, leading to a reduction of the resistance proportional to $m_{y}^{2}$ [5]. A similar dependence on the magnetization is found for the EMR, which is due to the combination of interfacial spinorbit scattering and spin-dependent conductivity of the FM [14-16].

Recent studies have shown that an additional currentdependent unidirectional magnetoresistance (UMR) emerges in FM/NM bilayers due to either the SHE or REE [8,9,21-24]. Unlike the most common magnetoresistive effects, including the anisotropic magnetoresistance (AMR), SMR, and EMR, the UMR is a nonlinear effect that violates Onsager reciprocity, being odd under either magnetization or current reversal. Because of its relationship to

spin-charge conversion and electron scattering phenomena, the UMR provides fundamental insight into the transport properties of spin-orbit coupled systems, including bulk crystals [25]. Moreover, owing to its unidirectional properties, the UMR can be used to electrically detect the sign of the magnetization in bilayer and multilayer samples using a simple two-terminal geometry $[8,24,26]$. Although it has been established that the UMR is a general property of FM/ NM [8,9,21-24], FM/semiconductor [26], and FM/topological insulator bilayers [27-29], its microscopic origin is still under debate.

Different mechanisms can give rise to UMR in FM/NM systems, even when considering a single source of spin accumulation such as the SHE. A first mechanism, sketched in Fig. 1(a), is the modulation of the interface resistance between the FM and NM due to the SHE-induced spin polarization, which changes the reflection and transmission coefficients of the electrons depending on the orientation of $\boldsymbol{\sigma}$ relative to $\mathbf{m}$ [8]. A second mechanism relies on the bulk spin-dependent conductivity of the FM, which again enhances or decreases the resistance of the FM/NM bilayer for

(a) Spin-dependent UMR

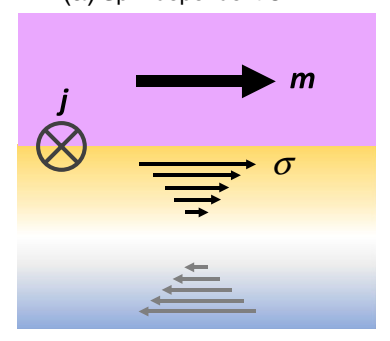

FIG. 1. (a) Illustration of the spin-dependent UMR and (b) spinflip UMR. 
parallel and antiparallel alignment of $\boldsymbol{\sigma}$ and $\mathbf{m}$ [30]. Both such mechanisms find a strong analogy with the current-in-plane giant magnetoresistance (GMR) in FM/NM/FM trilayers [31-34], where the role of one FM polarizer is replaced by the SHE in the NM, and differ from one another in the crucial role played by spin-dependent scattering occurring at the interface or in the bulk of the FM. A third mechanism, sketched in Fig. 1(b), invokes the creation or annihilation of magnons resulting from the absorption of the SHE-induced spin current in the FM [35]. In this case, the spin flips caused by electron-magnon scattering result in an increase or decrease, respectively, of the longitudinal resistance of the FM $[21,22,27]$. This last mechanism is related to the so-called spin-disorder resistivity of single FM conductors [36,37], which emerges also in the temperature and fielddependent measurements of thin films [38-41]. Note that these mechanisms differ from the nonlocal magnetoresistance recently reported in $\mathrm{Pt} / \mathrm{YIG}$ due to pure magnon currents $[42,43]$. In the following, we refer to the first and second mechanism as the interface and bulk spin-dependent (SD) UMR, respectively, and to the third as spin-flip (SF) UMR.

In this work, we investigate the origin of the UMR in FM/ NM metal layers as well as its current, magnetic field, and temperature dependence. We find that the three mechanisms described above coexist in $\mathrm{Co} / \mathrm{Pt}$ bilayers and that the SD-UMR and SF-UMR can be separated according to their different field and current dependence. Measurements of $\mathrm{Co}_{80} \mathrm{Cr}_{20} / \mathrm{Pt}$ further show that the interface and bulk spindependent scattering can be independently tuned to determine the sign and magnitude of the SD-UMR, similar to the direct and inverse GMR effect, whereas the SF-UMR depends on the temperature and magnon stiffness of the FM layer. Our results provide a unified picture of the microscopic processes leading to nonreciprocal electric transport in FM/NM conductors as well as practical insight on how to design heterostructures with tunable UMR.

We studied multilayer samples consisting of $\mathrm{Ta}(2.5) /$ $\mathrm{Co}(2.5) / \mathrm{Pt}(6) / \mathrm{Ta}(2) /$ substrate and $\mathrm{Ta}(2.5) / \mathrm{Co}_{80} \mathrm{Cr}_{20}$ $(1.6-5) / \mathrm{Pt}(4) / \mathrm{Ta}(2) /$ substrate grown on thermally oxidized $\mathrm{Si}$ wafers by magnetron sputtering (numbers in parentheses are thicknesses in $\mathrm{nm}$ ). The top Ta layer is naturally oxidized and nonconducting, and we assume that current shunting by the bottom Ta seed layer is negligible due to its high resistivity. All samples have in-plane magnetization. The blanket layers were patterned by optical lithography into Hall bars with lateral width $w=5,10 \mu \mathrm{m}$ and length $l \approx 4 w$. The magnetoresistance measurements were performed by applying an ac current $I=I_{0} \sin (\omega t)$ of frequency $\omega / 2 \pi=10 \mathrm{~Hz}$ and recording the first and second harmonic of the longitudinal voltage $V=V_{\omega}+$ $V_{2 \omega}=I R_{\omega}+I R_{2 \omega}$ as a function of $I$ and external magnetic field $B$ [8]. Here, the first harmonic $R_{\omega}$ represents the usual current-independent resistance of the bilayer, which includes contributions from the AMR, SMR, and EMR. The second harmonic $R_{2 \omega}(I)$ includes the different current-dependent
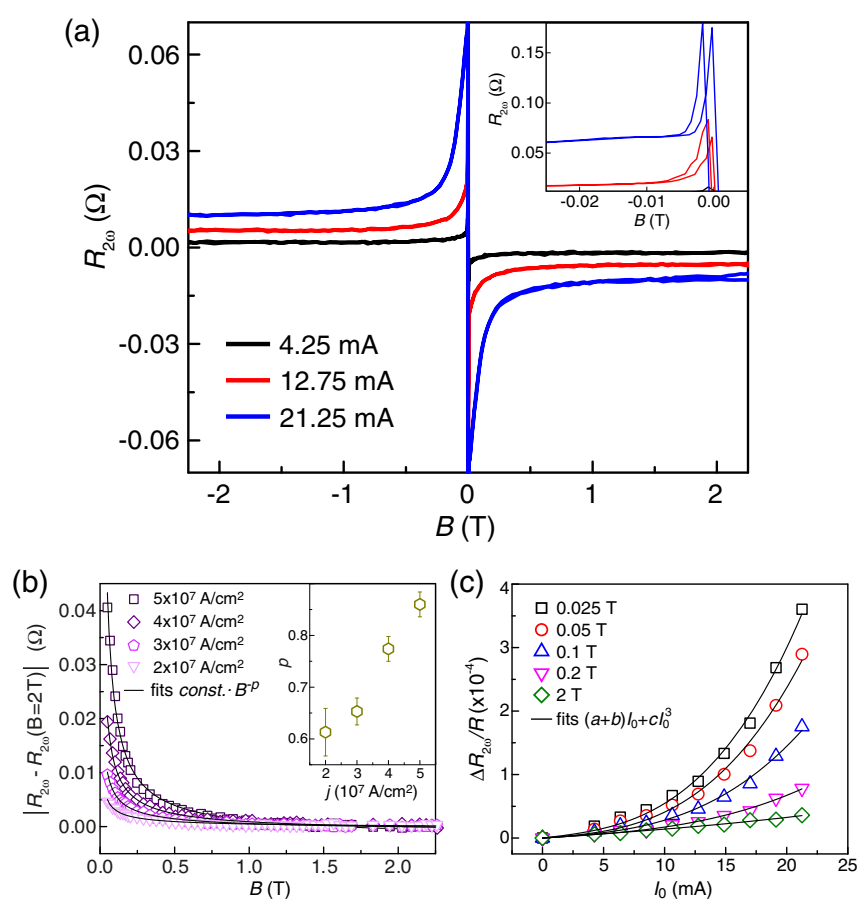

FIG. 2. (a) UMR of $\mathrm{Co}(2.5) / \mathrm{Pt}(6)$ as a function of magnetic field and current. Inset: detail of the low field region. (b) Fits of the field-dependent contribution to the UMR, $\mid R_{2 \omega}-R_{2 \omega}$ $(B=2 \mathrm{~T}) \mid \propto B^{-p}$, at different currents. Inset: dependence of the exponent $p$ on current. (c) Fits of the current dependence of the UMR at different fields.

contributions to the resistance, namely, the UMR, the changes of the magnetoresistance due to the oscillation of $\mathbf{m}$ induced by the spin-orbit torques [44], and the magnetothermal voltage induced by temperature gradients [45]. These contributions can be distinguished by their different symmetry and field dependence $[8,46]$. In $\mathrm{Co}(2.5) / \mathrm{Pt}(6)$, the magnetothermal voltage is less than $5 \%$ of the total signal and the spin-orbit torque-induced oscillations of the magnetoresistance are null for $\mathbf{m} \| \mathbf{y}$ [46]. In these conditions, $R_{2 \omega}$ represents the resistance difference between positive and negative current, namely, the UMR.

Figure 2(a) shows $R_{2 \omega}$ as a function of applied field $\mathbf{B} \| \mathbf{y}$ and $I$. The data evidence two distinct regimes, corresponding to low and high values of $B$. Above $1 \mathrm{~T}, R_{2 \omega}$ is dominated by a constant term that is independent of $B$ and proportional to $I$. This term is the SD-UMR previously reported by us and other groups $[8,9,23,26,30]$. Below $1 \mathrm{~T}$, on the other hand, $\left|R_{2 \omega}\right|$ increases sharply following a power law $\left|R_{2 \omega}(B, I)-R_{2 \omega}(2 T, I)\right| \propto B^{-p}$, with $p$ varying monotonically from 0.6 to 0.9 as a function of increasing current [Fig. 2(b)]. The increase of $R_{2 \omega}$ is even more remarkable near zero field [inset of Fig. 2(a)]. However, as the magnetization is not uniform and hysteretic in this limit, our analysis focuses on fields $|B|>0.02 \mathrm{~T}$. These data provide a first indication that different mechanisms simultaneously contribute to the UMR. In order to gain further insight into such mechanisms, we fit the relative resistance 
change $\Delta R_{2 \omega} / R=\left[R_{2 \omega}(\mathbf{m} \|+\mathbf{y})-R_{2 \omega}(\mathbf{m} \|-\mathbf{y})\right] / R$ with a polynomial function of the current [Fig. 2(c)]. We find excellent agreement for an expression of the type $[a+b(B)] I+c(B) I^{3}$, where $a$ is a constant independent of $B$, and $b$ and $c$ are two coefficients that scale inversely with $B$. This expression again supports the presence of two distinct scattering processes, one proportional to $a I$ and the other to $b(B) I+c(B) I^{3}$. Whereas the first term is consistent with the SD-UMR, the field dependence of the remaining terms indicates that the second process is related to the magnon population in the Co layer. It is well known that an applied field strongly reduces the magnon density in thin films, leading to a decrease of the resistance due to the reduction of thermal spin disorder [38]. Such an effect is clearly present also in our samples, and influences both $R_{\omega}$ [46] and $R_{2 \omega}$. We therefore attribute the decrease of the UMR in Figs. 2(a), 2(b) to the field counteracting the excitation of magnons by the spin current.

Further support for an electron-magnon scattering mechanism comes from the nonlinear current dependence shown in Fig. 2(c). Strong nonlinearities in the magnon population have been observed by Brillouin light scattering as the current intensity approaches the damping compensation threshold in FM/NM bilayers [35]. Together with Joule heating, such nonlinear effects determine the nonequilibrium density of magnons in the FM $[35,49]$, which ultimately affects $R_{2 \omega}$ due to spin-flip processes. In Co/Pt, our fits of the current dependence suggest that the spin current $(\propto I)$ modulates a thermalized magnon population $\propto T+\Delta T \propto b(B)+c(B) I^{2}$, where $T$ is the ambient temperature and $\Delta T \propto I^{2}$ is the temperature increase due to Joule heating [46]. Alternatively, a similar current dependence can be attributed to the direct excitation of lowfrequency magnons by the spin current [50]. We thus conclude that the UMR is given by the concurrence of spin-dependent and spin-flip scattering processes that have very different field and current dependencies. Whereas the SD-UMR dominates at high field, the SF-UMR produces the strongest magnetoresistance asymmetry at low field and high current. These results reconcile the interpretation of the UMR in terms of spin-dependent conductivity $[8,30]$ and magnon excitations [21,22,27].

Temperature- and angular-dependent measurements of $R_{2 \omega}$ offer further insight into the different properties of the SD-UMR and SF-UMR. In the Supplemental Material [46], we show that the SF-UMR decreases almost tenfold from 300 to $4 \mathrm{~K}$, whereas the SD-UMR decreases only twofold, highlighting the prominent role played by magnons in the first effect. Figure 3 shows the angular dependence of $R_{\omega}$ and $R_{2 \omega}$ measured at constant $B$ and $I$ while rotating the field in the $x y$ plane by an angle $\varphi$. We find that $R_{\omega}(\varphi)$ is proportional to $\sin ^{2} \varphi \propto m_{y}^{2}$ and not significantly affected by either $B$ or $I$, as expected for the SMR and AMR of a magnetically saturated layer [Figs. 3(a) and 3(c)]. On the other hand, $R_{2 \omega}(\varphi)$ varies strongly between low and high
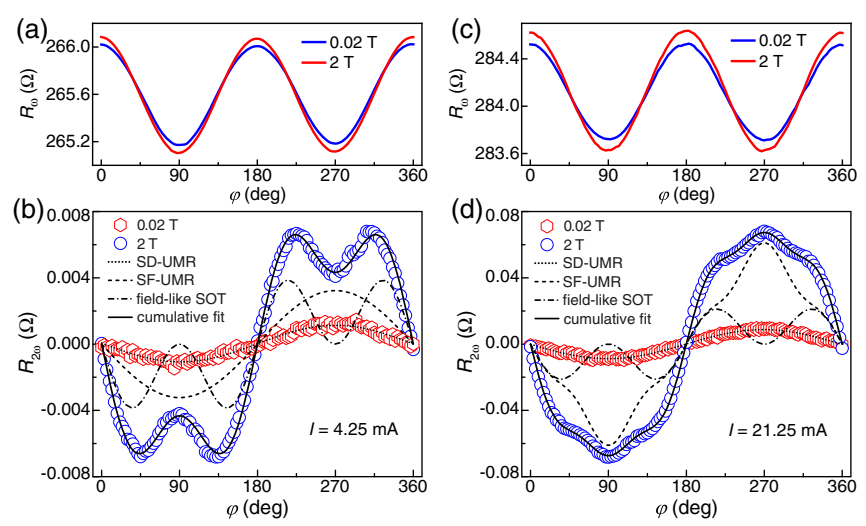

FIG. 3. Angular dependence of (a) $R_{\omega}$ and (b) $R_{2 \omega}$ at low current $\left(j=1 \times 10^{7} \mathrm{~A} / \mathrm{cm}^{2}\right)$ and (c),(d) high current $\left(j=5 \times 10^{7} \mathrm{~A} / \mathrm{cm}^{2}\right)$. The dotted, dot-dashed, and dashed lines are fits of the SD-UMR, spin-orbit torque, and SF-UMR contributions to $R_{2 \omega}$, respectively. See Ref. [46] for a description of the fit procedure.

field and also between low and high current. At high field both the spin-orbit torque and SF-UMR signals are small, and we observe the typical $\sin \varphi$ behavior expected of the SD-UMR [red symbols and dotted line in Figs. 3(b) and 3(d)]. At low current and low field [blue symbols in Fig. 3(b)] we observe four peaks at $\varphi=45^{\circ}, 135^{\circ}, 225^{\circ}$, $315^{\circ}$, which are characteristic of the fieldlike spin-orbit torque and Oersted field contribution to $R_{2 \omega}(\varphi)$ (dotdashed line) superimposed on the UMR $[8,45]$. At high current and low field [blue symbols in Fig. 3(d)], the SF-UMR signal is strongest, which results in two peaks at $\varphi=90^{\circ}$ and $270^{\circ}$ (dashed line). Interestingly, the high current SF-UMR is not simply proportional to $m_{y} \propto \sin \varphi$ as expected based on the product $\mathbf{m} \cdot \sigma$, but strongly peaked around $\varphi=90^{\circ}$ and $270^{\circ}$. Such a peaked angular dependence, which is even more evident for $\mathrm{Co}_{80} \mathrm{Cr}_{20} / \mathrm{Pt}$ [see Fig. 4], suggests that the magnon excitation probability becomes anisotropic as the current approaches the damping compensation threshold in the FM. We have presently no model for this effect, but note that such an anisotropy cannot be excluded on theoretical grounds [51] and that a peaked angular dependence has been reported also in the spin pumping signal of Pt/YIG bilayers [52].

Finally, we show that the SD-UMR consists of two separate contributions arising from bulk and interface spindependent scattering. In analogy with the GMR, we define a positive UMR when $\boldsymbol{\sigma}$ is parallel to the majority spins in the FM, resulting in a low resistance state. This situation is the most common and occurs, e.g., in $\mathrm{Co} / \mathrm{Pt}, \mathrm{Co} / \mathrm{Ta}$, and $\mathrm{Co} / \mathrm{W}$ bilayers, for which we have confirmed the sign of $\sigma$ by spin-orbit torque measurements. However, it is well known that a negative (inverse) GMR can be realized in magnetic multilayers in which the spin asymmetry coefficients for bulk $(\beta)$ and interface $(\gamma)$ scattering have opposite sign, such as $(\mathrm{FeCr}, \mathrm{FeV}, \mathrm{CoCr}) / \mathrm{Cu} / \mathrm{Co}[53,54]$. Because 

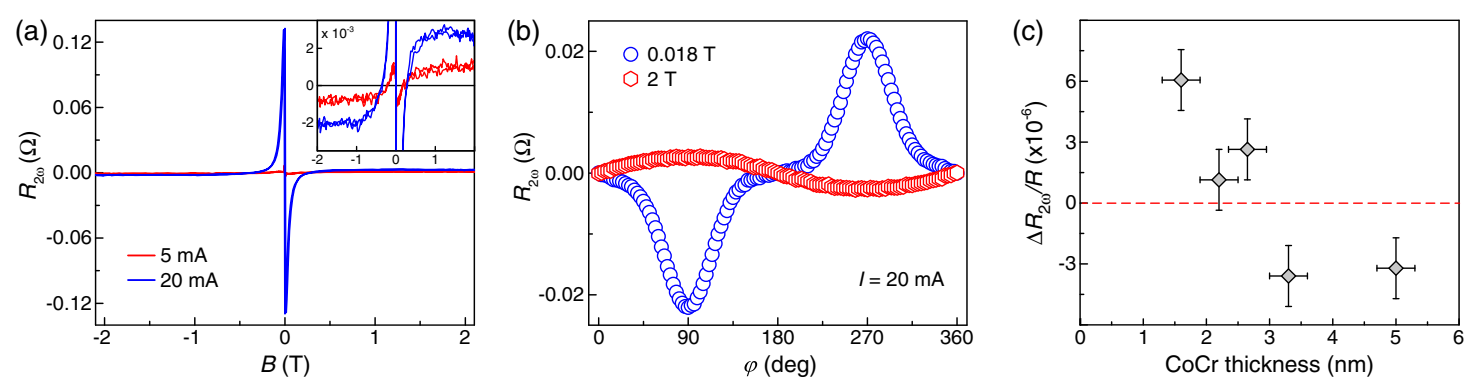

FIG. 4. (a) $\mathrm{UMR}$ of $\mathrm{Co}_{80} \mathrm{Cr}_{20}(3.3) / \mathrm{Pt}(4)$ as a function of magnetic field $B \| \mathbf{y}$. The curves show $R_{2 \omega}(B, I)$ measured at $I=5 \mathrm{~mA}$ $\left(j=0.7 \times 10^{7} \mathrm{~A} / \mathrm{cm}^{2}\right)$ and $20 \mathrm{~mA}\left(2.7 \times 10^{7} \mathrm{~A} / \mathrm{cm}^{2}\right)$. Inset: detail of the sign reversal of $R_{2 \omega}(B, I)$ as the negative SD-UMR prevails over the SF-UMR at high field. (b) Angular dependence of $R_{2 \omega}(B, I)$ at low and high field, representing the SF- and SD-UMR, respectively. (c) SD-UMR as a function of $\mathrm{Co}_{80} \mathrm{Cr}_{20}$ thickness measured at $B=2 \mathrm{~T}$ and $j=1 \times 10^{7} \mathrm{~A} / \mathrm{cm}^{2}$. The dashed line indicates the compensation of the bulk and interface spin-dependent scattering.

spin-dependent scattering underpins both phenomena [30], we expect that the magnitude and sign of the SD-UMR can be tuned in a similar way as the GMR. Further, by comparing the SD-UMR in systems with opposite sign of $\beta$ and $\gamma$, it should be possible to separately determine the bulk and interface contributions to the SD-UMR.

To test these hypotheses, we chose $\mathrm{Co}_{80} \mathrm{Cr}_{20}$ as a model FM in which the conductivity of the minority electrons is larger than that of the majority electrons, i.e., $\beta<0$ [54], and measured the UMR of $\mathrm{Co}_{80} \mathrm{Cr}_{20} / \mathrm{Pt}(4)$ bilayers of different thickness. Figure 4(a) shows the $R_{2 \omega}$ of $\mathrm{Co}_{80} \mathrm{Cr}_{20}(3.3) / \mathrm{Pt}(4)$. Similar to the measurements reported in Fig. 2(c), we observe that $R_{2 \omega}$ is significantly enhanced at high current and low field. This enhancement arises from the SF-UMR, which has the same sign as in Co/Pt. In contrast to $\mathrm{Co} / \mathrm{Pt}$, however, $R_{2 \omega}$ changes sign above $0.15 \mathrm{~T}$, becoming negative in the high field regime dominated by the SD-UMR. The sign reversal is confirmed by the angular-dependent measurements of $R_{2 \omega}$ performed at fields representative of the SF-UMR and SD-UMR regimes [Fig. 4(b)]. We thus focus on the high field behavior of $R_{2 \omega}$ in $\mathrm{Co}_{80} \mathrm{Cr}_{20}$ to investigate the sign change of the SD-UMR. Figure 4(c) reports $\Delta R_{2 \omega}(2 T) / R$ as a function of $\mathrm{Co}_{80} \mathrm{Cr}_{20}$ thickness after subtraction of the magnetothermal signal [46]. The relatively large error bars are due to low signalto-noise ratio, uncertainties in the separation of the magnetothermal voltage, and thickness variations along the $\mathrm{Co}_{80} \mathrm{Cr}_{20}$ wedge. We observe that $\Delta R_{2 \omega}(2 T) / R$ is positive below $\sim 3 \mathrm{~nm}$, similar to $\mathrm{Co} / \mathrm{Pt}$, and negative above. The existence of a compensation thickness with zero UMR unambiguously demonstrates that $\beta$ and $\gamma$ have opposite sign in $\mathrm{Co}_{80} \mathrm{Cr}_{20} / \mathrm{Pt}$ and that the SD-UMR of the thicker films is determined by bulk spin-dependent scattering with $\beta<0$. Such a behavior is reminiscent of the GMR inversion in $\mathrm{Co}_{80} \mathrm{Cr}_{20} / \mathrm{Cu} / \mathrm{Co}$ multilayers [54], which leads us to conclude that there are two competing contributions to the SD-UMR: one due to interface scattering, which is generally positive $(\gamma>0)$ and prevails in the limit of thin FM, and one due to bulk scattering, which can be either positive $(\beta>0)$ or negative $(\beta<0)$ and dominates in thick FM.

A corollary to these measurements is that the SF-UMR has the same sign in $\mathrm{Co}_{80} \mathrm{Cr}_{20} / \mathrm{Pt}$ as in $\mathrm{Co} / \mathrm{Pt}$, independently of thickness. This result can be easily explained by considering that the direction of the spin-orbit torques and magnetization remain the same in the two systems, such that the combination of current and magnetization required for exciting or annihilating magnons does not change. Interestingly, however, the field-induced damping of the SF-UMR does depend on the $\mathrm{Co}_{80} \mathrm{Cr}_{20}$ thickness. For this system, we find that $\left|R_{2 \omega}(B, I)-R_{2 \omega}(2 T, I)\right| \propto B^{-p}$, with $p$ dropping from 1.7 to 1.1 in 2 and $5 \mathrm{~nm}$ thick $\mathrm{Co}_{80} \mathrm{Cr}_{20}$ films, respectively [46]. Such a drop may be attributed to the increase in magnon stiffness that occurs in FM films as spin disorder progressively reduces with increasing thickness [40]. This behavior provides additional evidence that the SF-UMR and SD-UMR originate from distinct phenomena and can be separately controlled by modifying the composition and thickness of the FM layer.

In summary, we have shown that three different mechanisms determine the UMR of metal bilayers, namely, the bulk and interface SD-UMR and the SF-UMR. These mechanisms can be separated by their distinct field and current dependence. Whereas the SD-UMR is independent of $B$ and proportional to $j$, the SF-UMR scales with $B^{-p}$ and is proportional to $j+j^{3}$. The monotonic field dependence of the SF-UMR originates from the field-induced gap in the magnon excitation spectrum, which quenches the electron-magnon scattering at high field. The exponent of the power law $B^{-p}$ increases with current and decreases with the thickness of the FM [46], as expected for the softening of the magnon modes with temperature due to Joule heating and stiffening of the modes with thickness, respectively. Another prominent difference between the SF-UMR and SD-UMR is that the former is always positive, whereas the latter can be either positive or negative. The positive SD-UMR of $\mathrm{Co} / \mathrm{Pt}$ concords with the positive spin asymmetry coefficients for bulk and interface scattering of 
Co and $\mathrm{Co} / \mathrm{Pt}$, respectively, as determined by GMR $[54,55]$. Measurements of $\mathrm{Co}_{80} \mathrm{Cr}_{20} / \mathrm{Pt}$, on the other hand, show that the SD-UMR becomes negative when $\mathrm{Co}_{80} \mathrm{Cr}_{20}$ is thicker than $3 \mathrm{~nm}$. This behavior is similar to the inverse GMR effect, which indicates that both the interface and bulk SD-UMR are present and have opposite sign. The possibility of tuning the UMR by modifying the magnon spectrum as well as the relative weight of bulk and interface electron scattering makes this phenomenon very appealing to study electron transport in spin-orbit coupled systems as well as to measure the magnetization in two-terminal devices.

We acknowledge fruitful discussions with S.O. Demokritov. This work was supported by the Swiss National Science Foundation (Grant No. 200020-172775) and by C-SPIN, one of the six SRC STARnet Centers, sponsored by MARCO and DARPA.

[1] A. Manchon, I. M. Miron, T. Jungwirth, J. Sinova, J. Zelezný, A. Thiaville, K. Garello, and P. Gambardella, Current-induced spin-orbit torques in ferromagnetic and antiferromagnetic systems, arXiv:1801.09636.

[2] H. Nakayama, M. Althammer, Y.-T. Chen, K. Uchida, Y. Kajiwara, D. Kikuchi, T. Ohtani, S. Geprägs, M. Opel, S. Takahashi, R. Gross, G. E. W. Bauer, S. T. B. Goennenwein, and E. Saitoh, Spin Hall Magnetoresistance Induced by a Nonequilibrium Proximity Effect, Phys. Rev. Lett. 110, 206601 (2013).

[3] M. Althammer et al., Quantitative study of the spin Hall magnetoresistance in ferromagnetic insulator/normal metal hybrids, Phys. Rev. B 87, 224401 (2013).

[4] N. Vlietstra, J. Shan, V. Castel, B. J. van Wees, and J. B. Youssef, Spin-Hall magnetoresistance in platinum on yttrium iron garnet: Dependence on platinum thickness and in-plane/out-of-plane magnetization, Phys. Rev. B 87, 184421 (2013).

[5] Y.-T. Chen, S. Takahashi, H. Nakayama, M. Althammer, S. T. B. Goennenwein, E. Saitoh, and G. E.W. Bauer, Theory of spin Hall magnetoresistance, Phys. Rev. B 87, 144411 (2013).

[6] B. F. Miao, S. Y. Huang, D. Qu, and C. L. Chien, Physical Origins of the New Magnetoresistance in Pt/YIG, Phys. Rev. Lett. 112, 236601 (2014).

[7] M. Isasa, A. Bedoya-Pinto, S. Vélez, F. Golmar, F. Sánchez, L. E. Hueso, J. Fontcuberta, and F. Casanova, Spin Hall magnetoresistance at $\mathrm{Pt} / \mathrm{CoFe} 2 \mathrm{O} 4$ interfaces and texture effects, Appl. Phys. Lett. 105, 142402 (2014).

[8] C. O. Avci, K. Garello, A. Ghosh, M. Gabureac, S. F. Alvarado, and P. Gambardella, Unidirectional spin Hall magnetoresistance in ferromagnet/normal metal bilayers, Nat. Phys. 11, 570 (2015).

[9] C. O. Avci, K. Garello, J. Mendil, A. Ghosh, N. Blasakis, M. Gabureac, M. Trassin, M. Fiebig, and P. Gambardella, Magnetoresistance of heavy and light metal/ferromagnet bilayers, Appl. Phys. Lett. 107, 192405 (2015).

[10] J. Kim, P. Sheng, S. Takahashi, S. Mitani, and M. Hayashi, Spin Hall Magnetoresistance in Metallic Bilayers, Phys. Rev. Lett. 116, 097201 (2016).
[11] M. I. Dyakonov, Magnetoresistance due to Edge Spin Accumulation, Phys. Rev. Lett. 99, 126601 (2007).

[12] S. Velez, V. N. Golovach, A. Bedoya-Pinto, M. Isasa, E. Sagasta, M. Abadia, C. Rogero, L. E. Hueso, F. S. Bergeret, and F. Casanova, Hanle Magnetoresistance in Thin Metal Films with Strong Spin-Orbit Coupling, Phys. Rev. Lett. 116, 016603 (2016).

[13] A. Kobs, S. Heße, W. Kreuzpaintner, G. Winkler, D. Lott, P. Weinberger, A. Schreyer, and H. P. Oepen, Anisotropic Interface Magnetoresistance in $\mathrm{Pt} / \mathrm{Co} / \mathrm{Pt}$ Sandwiches, Phys. Rev. Lett. 106, 217207 (2011).

[14] V. L. Grigoryan, W. Guo, G. E. W. Bauer, and J. Xiao, Intrinsic magnetoresistance in metal films on ferromagnetic insulators, Phys. Rev. B 90, 161412 (2014).

[15] S. S.-L. Zhang and S. Zhang, Angular dependence of anisotropic magnetoresistance in magnetic systems, J. Appl. Phys. 115, 17C703 (2014).

[16] S. S.-L. Zhang, G. Vignale, and S. Zhang, Anisotropic magnetoresistance driven by surface spin-orbit scattering, Phys. Rev. B 92, 024412 (2015).

[17] T. Hupfauer, A. Matos-Abiague, M. Gmitra, F. Schiller, J. Loher, D. Bougeard, C. H. Back, J. Fabian, and D. Weiss, Emergence of spin-orbit fields in magnetotransport of quasitwo-dimensional iron on gallium arsenide, Nat. Commun. 6, 7374 (2015).

[18] H. Nakayama, Y. Kanno, H. An, T. Tashiro, S. Haku, A. Nomura, and K. Ando, Rashba-Edelstein Magnetoresistance in Metallic Heterostructures, Phys. Rev. Lett. 117, 116602 (2016).

[19] H. Nakayama, H. An, A. Nomura, Y. Kanno, S. Haku, Y. Kuwahara, H. Sakimura, and K. Ando, Temperature dependence of Rashba-Edelstein magnetoresistance in $\mathrm{Bi} / \mathrm{Ag} / \mathrm{CoFeB}$ trilayer structures, Appl. Phys. Lett. 110, 222406 (2017).

[20] J. Kim, Y.-T. Chen, S. Karube, S. Takahashi, K. Kondou, G. Tatara, and Y.C. Otani, Evaluation of bulk-interface contributions to Edelstein magnetoresistance at metal/oxide interfaces, Phys. Rev. B 96, 140409 (2017).

[21] S. Langenfeld, V. Tshitoyan, Z. Fang, A. Wells, T. A. Moore, and A.J. Ferguson, Exchange magnon induced resistance asymmetry in permalloy spin-hall oscillators, Appl. Phys. Lett. 108, 192402 (2016).

[22] T. Li, S. Kim, S.-J. Lee, S.-W. Lee, T. Koyama, D. Chiba, T. Moriyama, K.-J. Lee, K.-J. Kim, and T. Ono, Origin of threshold current density for asymmetric magnetoresistance in Pt/Py bilayers, Appl. Phys. Express 10, 073001 (2017).

[23] Y. Yin, D.-S. Han, M. C. H. de Jong, R. Lavrijsen, R. A. Duine, H. J. M. Swagten, and B. Koopmans, Thickness dependence of unidirectional spin-hall magnetoresistance in metallic bilayers, Appl. Phys. Lett. 111, 232405 (2017).

[24] C. O. Avci, M. Mann, A. J. Tan, P. Gambardella, and G.S.D. Beach, A multi-state memory device based on the unidirectional spin hall magnetoresistance, Appl. Phys. Lett. 110, 203506 (2017).

[25] T. Ideue, K. Hamamoto, S. Koshikawa, M. Ezawa, S. Shimizu, Y. Kaneko, Y. Tokura, N. Nagaosa, and Y. Iwasa, Bulk rectification effect in a polar semiconductor, Nat. Phys. 13, 578 (2017). 
[26] K. Olejník, V. Novák, J. Wunderlich, and T. Jungwirth, Electrical detection of magnetization reversal without auxiliary magnets, Phys. Rev. B 91, 180402 (2015).

[27] K. Yasuda, A. Tsukazaki, R. Yoshimi, K. S. Takahashi, M. Kawasaki, and Y. Tokura, Large Unidirectional Magnetoresistance in a Magnetic Topological Insulator, Phys. Rev. Lett. 117, 127202 (2016).

[28] K. Yasuda, A. Tsukazaki, R. Yoshimi, K. Kondou, K. S. Takahashi, Y Otani, M. Kawasaki, and Y. Tokura, CurrentNonlinear Hall Effect and Spin-Orbit Torque Magnetization Switching in a Magnetic Topological Insulator, Phys. Rev. Lett. 119, 137204 (2017).

[29] Y. Lv, J. Kally, D. Zhang, J. S. Lee, M. Jamali, N. Samarth, and J.-P. Wang, Unidirectional spin-hall and rashba- edelstein magnetoresistance in topological insulator-ferromagnet layer heterostructures, Nat. Commun. 9, 111 (2018).

[30] S. S.-L. Zhang and G. Vignale, Theory of unidirectional spin hall magnetoresistance in heavy-metal/ferromagneticmetal bilayers, Phys. Rev. B 94, 140411 (2016).

[31] M. N. Baibich, J. M. Broto, A. Fert, F. N. Van Dau, F. Petroff, P. Eitenne, G. Creuzet, A. Friederich, and J. Chazelas, Giant Magnetoresistance of (001)Fel(001)Cr Magnetic Snperlattices, Phys. Rev. Lett. 61, 2472 (1988).

[32] G. Binasch, P. Grunberg, F. Saurenbach, and W. Zinn, Enhanced magnetoresistance in layered magnetic structures with antiferromagnetic interlayer exchange, Phys. Rev. B 39, 4828(R) (1989).

[33] R. E. Camley and J. Barnaś, Theory of Giant Magnetoresistance Effects in Magnetic Layered Structures with Antiferromagnetic Coupling, Phys. Rev. Lett. 63, 664 (1989).

[34] R. Q. Hood and L. M. Falicov, Boltzmann-equation approach to the negative magnetoresistance of ferromagneticnormal-metal multilayers, Phys. Rev. B 46, 8287 (1992).

[35] V. E. Demidov, S. Urazhdin, E. R. J. Edwards, M. D. Stiles, R. D. McMichael, and S. O. Demokritov, Control of Magnetic Fluctuations by Spin Current, Phys. Rev. Lett. 107, 107204 (2011).

[36] T. Kasuya, Electrical resistance of ferromagnetic metals, Prog. Theor. Phys. 16, 58 (1956).

[37] D. A. Goodings, Electrical resistivity of ferromagnetic metals at low temperatures, Phys. Rev. 132, 542 (1963).

[38] B. Raquet, M. Viret, E. Sondergard, O. Cespedes, and R. Mamy, Electron-magnon scattering and magnetic resistivity in 3d ferromagnets, Phys. Rev. B 66, 024433 (2002).

[39] A. P. Mihai, J. P. Attané, A. Marty, P. Warin, and Y. Samson, Electron-magnon diffusion and magnetization reversal detection in FePt thin films, Phys. Rev. B 77, 060401 (2008).

[40] R. M. Rowan-Robinson, A. T. Hindmarch, and D. Atkinson, Enhanced electron-magnon scattering in ferromagnetic thin films and the breakdown of the Mott two-current model, Phys. Rev. B 90, 104401 (2014).

[41] Y. Cheng, K. Chen, and S. Zhang, Interplay of magnon and electron currents in magnetic heterostructure, Phys. Rev. B 96, 024449 (2017).

[42] L. J. Cornelissen, J. Liu, R. A. Duine, J. B. Youssef, and B. J. Van Wees, Long-distance transport of magnon spin information in a magnetic insulator at room temperature, Nat. Phys. 11, 1022 (2015).
[43] S. T. B. Goennenwein, R. Schlitz, M. Pernpeintner, K. Ganzhorn, M. Althammer, R. Gross, and H. Huebl, Nonlocal magnetoresistance in YIG/Pt nanostructures, Appl. Phys. Lett. 107, 172405 (2015).

[44] K. Garello, I. M. Miron, C. O. Avci, F. Freimuth, Y. Mokrousov, S. Blügel, S. Auffret, O. Boulle, G. Gaudin, and P. Gambardella, Symmetry and magnitude of spin-orbit torques in ferromagnetic heterostructures, Nat. Nanotechnol. 8, 587 (2013).

[45] C. O. Avci, K. Garello, M. Gabureac, A. Ghosh, A. Fuhrer, S. F. Alvarado, and P. Gambardella, Interplay of spin-orbit torque and thermoelectric effects in ferromagnet/normalmetal bilayers, Phys. Rev. B 90, 224427 (2014).

[46] See Supplemental Material at http://link.aps.org/ supplemental/10.1103/PhysRevLett.121.087207 for a description of the harmonic analysis of the nonlinear magnetoresistance, spin disorder magnetoresistance, temperature dependent measurements, and fits, which includes Refs. [47,48].

[47] V. P. Amin and M. D. Stiles, Spin transport at interfaces with spin-orbit coupling: Phenomenology, Phys. Rev. B 94, 104420 (2016).

[48] T. D. Skinner, M. Wang, A. T. Hindmarch, A. W. Rushforth, A. C. Irvine, D. Heiss, H. Kurebayashi, and A. J. Ferguson, Spin-orbit torque opposing the Oersted torque in ultrathin Co/Pt bilayers, Appl. Phys. Lett. 104, 062401 (2014).

[49] T. Meyer, T. Brächer, F. Heussner, A. A. Serga, H. Naganuma, K. Mukaiyama, M. Oogane, Y. Ando, B. Hillebrands, and P. Pirro, Experimental investigation of the temperature-dependent magnon density and its influence on studies of spin-transfer-torque-driven systems, IEEE Magn. Lett. 8, 3108005 (2017).

[50] I. V. Borisenko, V. E. Demidov, S. Urazhdin, A. B. Rinkevich, and S. O. Demokritov, Relation between unidirectional spin hall magnetoresistance and spin current-driven magnon generation, Appl. Phys. Lett. 113, 062403 (2018).

[51] S. M. Rezende, F. M. de Aguiar, and A. Azevedo, Spin-Wave Theory for the Dynamics Induced by Direct Currents in Magnetic Multilayers, Phys. Rev. Lett. 94, 037202 (2005).

[52] M. Collet, X. de Milly, O. d'Allivy Kelly, V. V. Naletov, R. Bernard, P. Bortolotti, J. B. Youssef, V. E. Demidov, S. O. Demokritov, J. L. Prieto, M. Muñoz, V. Cros, A. Anane, G. de Loubens, and O. Klein, Generation of coherent spinwave modes in yttrium iron garnet microdiscs by spin-orbit torque, Nat. Commun. 7, 10377 (2016).

[53] J. M. George, L. G. Pereira, A. Barthélémy, F. Petroff, L. Steren, J. L. Duvail, A. Fert, R. Loloee, P. Holody, and P. A. Schroeder, Inverse Spin-Valve-Type Magnetoresistance in Spin Engineered Multilayered Structures, Phys. Rev. Lett. 72, 408 (1994).

[54] C. Vouille, A. Barthélémy, F. E. Mpondo, A. Fert, P. A. Schroeder, S. Y. Hsu, A. Reilly, and R. Loloee, Microscopic mechanisms of giant magnetoresistance, Phys. Rev. B 60, 6710 (1999).

[55] H. Y. T. Nguyen, W. P. Pratt, and J. Bass, Spin-flipping in Pt and at $\mathrm{Co} / \mathrm{Pt}$ interfaces, J. Magn. Magn. Mater. 361, 30 (2014). 